\title{
A Brain-Computer Interface for High-Level Remote Control of an Autonomous, Reinforcement-Learning-Based Robotic System for Reaching and Grasping
}

\author{
Thomas Lampe ${ }^{* \ddagger}$ \\ tlampe@informatik.uni- \\ freiburg.de \\ Alexander Knorr ${ }^{\dagger}$ \\ alexander.knorr@uniklinik- \\ freiburg.de
}

\author{
Lukas D. J. Fiederer ${ }^{\dagger \ddagger}$ \\ lukas.fiederer@uniklinik- \\ freiburg.de \\ Martin Riedmiller* \\ riedmiller@informatik.uni- \\ freiburg.de
}

\author{
Martin Voelker ${ }^{\dagger}$ \\ martin.voelker@uniklinik- \\ freiburg.de \\ Tonio Ball ${ }^{\dagger}$ \\ tonio.ball@uniklinik- \\ freiburg.de
}

\begin{abstract}
We present an Internet-based brain-computer interface (BCI) for controlling an intelligent robotic device with autonomous reinforcement-learning. BCI control was achieved through dry-electrode electroencephalography (EEG) obtained during imaginary movements. Rather than using low-level direct motor control, we employed a high-level control scheme of the robot, acquired via reinforcement learning, to keep the users cognitive load low while allowing control a reachinggrasping task with multiple degrees of freedom. High-level commands were obtained by classification of EEG responses using an artificial neural network approach utilizing timefrequency features and conveyed through an intuitive user interface. The novel combination of a rapidly operational dry electrode setup, autonomous control and Internet connectivity made it possible to conveniently interface subjects in an EEG laboratory with remote robotic devices in a closed-loop setup with online visual feedback of the robots actions to the subject. The same approach is also suitable to provide homebound patients with the possibility to control state-of-the-art robotic devices currently confined to a research environment. Thereby, our BCI approach could help severely paralyzed patients by facilitating patient-centered research of new means of communication, mobility and independence.
\end{abstract}

\section{Author Keywords}

Semi-autonomous systems; Robots; Camera-based UIs; Machine Learning and Data Mining

\section{ACM Classification Keywords}

H.5.2. Information Interfaces and Presentation (e.g. HCI): User Interfaces

\footnotetext{
*Albert-Ludwigs-University Freiburg, Machine Learning Lab, Georges-Köhler-Allee 79, 79110 Freiburg, Germany

${ }^{\dagger}$ Albert-Ludwigs-University Freiburg, iEEG and Brain Imaging Group, Engelbergerstr. 21, 79108 Freiburg, Germany

${ }^{\ddagger}$ These authors contributed equally.

Permission to make digital or hard copies of part or all of this work for personal or classroom use is granted without fee provided that copies are not made or distributed for profit or commercial advantage and that copies bear this notice and the full citation on the first page. Copyrights for third-party components of this work must be honored. For all other uses, contact the Owner/Author.

Copyright is held by the owner/author(s).

IUI'14, Feb 24-27 2014, Haifa, Israel

ACM 978-1-4503-2184-6/14/02.

http://dx.doi.org/10.1145/2557500.2557533
}

\section{General Terms}

Human Factors; Experimentation

\section{INTRODUCTION}

The use of brain-computer interfaces (BCIs) for robot control has become an integral field of research in recent years, intimately blending neuroscience and robotics. One central application that has received much attention is the control of prosthetic actuators by disabled individuals. Systems have developed far enough to enable tetraplegic patients to interact with the world $[4,7]$ by translating neuronal activity measured in the motor cortex into control signals steering external effectors such as a robotic device.

While the relevance and clinical promise of such work can hardly be emphasized enough, one drawback of BCIs relying on low-level motor control such as during reaching-grasping tasks is the limited accuracy that can be achieved with current $\mathrm{BCI}$ techniques, even after extensive training. To ameliorate the problem of limited accuracy, cognitive BCI approaches combined with autonomous intelligent systems have been proposed [12].

In this framework, a user should ideally only have to control the system on a higher level, the intuitive vision being that one would only need to think of what to do, not how to do it. The actuator would then perform the detailed action by itself to achieve the specified goal. Such a high-level approach would reduce the cognitive load required of the user. As a result, semi-autonomous systems would be intuitive to use and might be adapted to more quickly.

Here, we developed and tested an intuitive user interface that can be controlled via electroencephalography (EEG) signals to perform a reaching-grasping task carried out by an autonomous, reinforcement-learning-based robotic system. Motor-imagery-related EEG changes classified in single trials were used to select the target object to be grasped. An autonomous robotic arm then performed the desired action without the need for low-level control by the user.

While previous BCI research has to a large extent focused on improving recording and decoding techniques and has made tremendous progress in these fields, relatively little work so far has been devoted to the development and refinement of dedicated user interfaces for experimental and emerging clin- 
ical BCI applications [1]. Here, we present a user interface that is based on automatic image segmentation to identify graspable objects within the range of the robotic arm. In the video stream presented to the subjects, graspable objects are overlaid with a selection box that can be moved among the set of identified objects based on BCI commands issued by the user.

On the control side, we made use of reinforcement learning (RL) [18] to acquire most of the needed grasping skills only through trial-and-error. While not strictly necessary for the principal idea of an intelligent prosthesis, such an approach is desirable, as it allows the adaption of the system to changes in the environment or in the robot itself. Furthermore, this approach does not require engineering of the control skills. Thus, rather than merely shifting the learning required from the user to an expert programmer or engineer, we leave it entirely to the machine.

On the EEG side, our emphasis was on testing a comfortable and quickly usable setup, rather than optimizing decoding accuracy. Particularly, by using dry EEG electrodes, which are attached fast and easily and do not need electrode gel or scalp scraping, we avoided exposing subjects and patients to an uncomfortable situation. While the present dry electrode-based EEG setup is not meant to replace such approaches aimed at high accuracy, it proved to be useful for extensive testing and optimization of the user interface.

The novelty of our approach lies in the combination of camera-based visual servoing control with reinforcement learning in a brain-computer interface. Additionally, we used dry electrodes for EEG control based on multi-class decoding, instead of wet electrodes as typically used in similar previous EEG-based BCIs. To the best of our knowledge, no dry electrodes have been used before for a system that is as quick to set up while allowing detection of four motor imagery classes, and no such system has incorporated reinforcement learning, which adds extra potential for user adaptability. Other remote BCI systems were also mostly aimed at mobile navigation (ex. [5]). Some works have used an arm for manipulation, but either involved an SSVEP interface rather than one based on motor imagery [3] or employed direct kinematic motor control for fewer degrees of freedom (DOFs) [6].

The feasibility of robot control was demonstrated in three subjects. In addition, a larger study with screen cursor instead of robot control was performed in order to further quantify the reliability of the dry-electrode-based EEG control.

\section{SYSTEM ARCHITECTURE}

The general setup we propose assumes that a user is attached to EEG hardware. A semi-autonomous robotic device is to be controlled remotely while situated at another location.

As an example of such a setup, we considered the case of the Jaco robotic arm (Kinova, Montreal, Canada), capable of grasping colored balls in its working area. Instead of the arm being controlled directly, the user merely needed to choose the specific object to be manipulated through high-level EEG commands. Once an object had been chosen, it was autonomously picked up through the behaviors elaborated on in

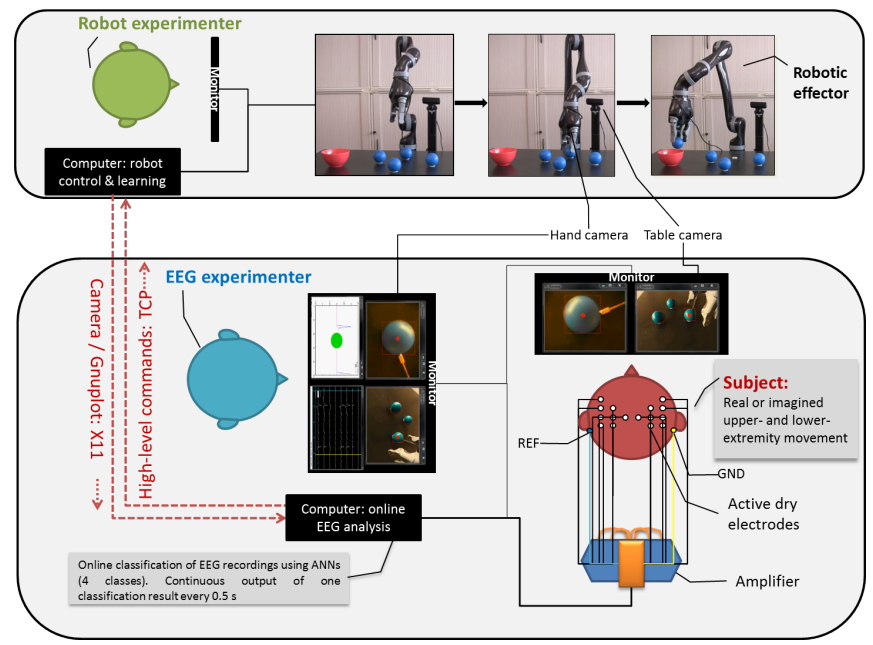

Figure 1. Schematic representation of experimental setup. Subjects and robot are located several kilometers apart and connected through the Internet by TCP/IP and X-forwarding. EEG Subjects see the online video stream of hand and table cameras of the robot arm. The EEG experimenter sees both video streams, the sent data and the ongoing EEG recordings. The robot experimenter sees both video streams, the received data and the flow of robotic control. GND: ground; REF: reference; ANN: artificial neural network.

the next section. An overview of the architecture is illustrated in Figure 1.

Users were presented with two webcam views, one showing a hand view and the other a scene view (c.f. Figure $2 c \& b$, respectively). Objects recognized in either camera were framed with selection boxes. Three types of BCI events which conceptually corresponded to left, right and confirmation were then used to select the desired target from these objects. Left and right signals were forwarded directly to the vision module, which selected the object next to the currently tracked one in the given direction. In this way, a user could cycle through available targets. Confirmation events were processed by the control system itself, and cause it to toggle through a series of process stages.

Initially, the arm was in a waiting position, with the user selecting the object to grasp in the table camera's view. A first confirmation triggered the actuator's approach towards a position located close to the target. The view then switched to the hand-mounted camera to allow the user to choose again between multiple close objects or to amend incorrect choices in the first attempt before performing the actual grasp. Once ready, a second confirmation started the short-range controller that grasps the object and picks it up. Then, the user would issue two more confirmation events, launching handcoded feed-forward behaviors that moved the arm over a drop point and opened the gripper, respectively, before the cycle restarted.

Communication between decoder and robot controller was realized via a custom TCP/IP interface. Here, the commands sent to the controller were strings of either $\left[\begin{array}{ll}0 & 0\end{array}\right]$ for no action, [ [ 1 1] for right, $\left[\begin{array}{ll}0 & -1\end{array}\right]$ for left, or [1 0$]$ for confirmation. These specific commands were ultimately arbitrary and easily exchanged or extended. The numeric coding was used 


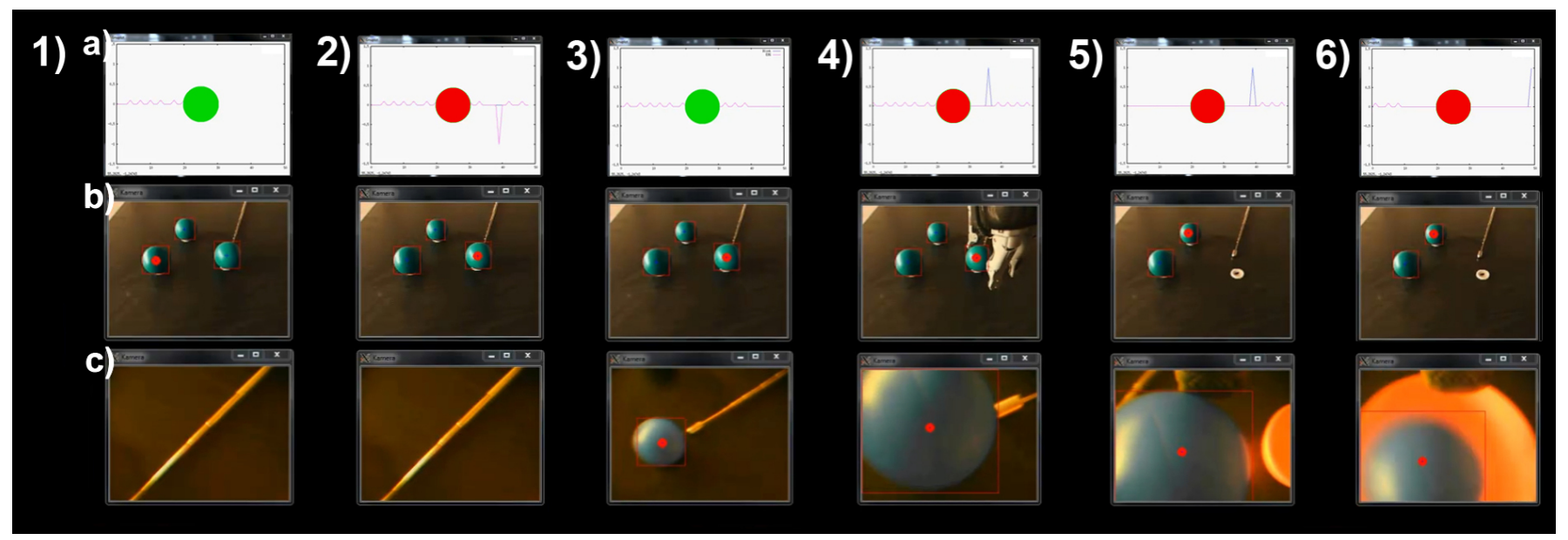

Figure 2. Windows of the control interface. Subjects see (b) \& (c), both experimenters see all three windows. (a) Overview of recognized events and lock status during the last 5 seconds: Blue spikes indicate recent confirmation events, red spikes pointing downwards or upwards imply movement of the selection box to the next or preceding ball in the sequence, respectively. Small red spikes and the red circle indicate the lock state. (b) Desk cameras view. The target object to be grasped is indicated by a metal pointer. (c) View from camera inside the robotic hand. 1) Start, the user is supposed to pick up the ball on the bottom right as indicated by the pointer. 2) Decoding of right hand movement, cursor in the desk camera (b) jumps to the right. 3) Decoding of toe movement, i.e., a conformation event; the robotic arm moves toward the target ball, control switches to the hand camera (c). 4) Confirmation event causes the robotic arm to pick up the ball. 5) Another conformation event lets the robotic hand to move over a bowl, and a last conformation in 6) causes the robotic hand drop the ball.

here simply for ease of processing on the robotic side, which needed to transform any command into such a sequence for its internal representation. In principle, any high-level command string could have been be used, such as look_left. After each command, the locking signal [0.1 0.1$]$ was sent to prevent sending commands multiple times. Sent commands were displayed online to the user for greater feedback (Figure 2). This graph, as well as the live camera streams, were accessed on the users side via X11-forwarding.

\section{ROBOT CONTROL}

The physical system was centered around a Kinova Jaco robotic arm, a therapeutic model commonly used by individuals capable of using their hands but lacking arm strength, and designed for installation on a wheelchair.

The autonomous control of this robotic system followed an identical approach to that described in previous work [8]. Two separate modules were responsible for the actual grasping behavior of the arm. The first, dubbed the long-range controller, realized a coarse mapping to bring the actuator close to a target object using the camera mounted on the table. It was therefore activated after the initial target selection by the user. In the second stage, a more precise short-range controller took over using a visual servoing approach to perform the fine movements needed for a secure grasp.

To train the autonomous agent, techniques of both supervised and reinforcement learning were used. The network that constituted the long-range controller was trained in a supervised fashion, i.e. by recording sets of input and desired output. Specifically, it realized a mapping from object locations in the stationary camera to kinematic positions of the actuator in the horizontal plane. In contrast, the short-range controller was trained using reinforcement learning. Thus the system learned an ideal grasp policy by interacting with the environ- ment and learning from success and error. The arm started at a random position over the working area, with the likewise randomly placed object being visible in the hand camera. Episodes alternated between greedy exploitation of the current policy and $\epsilon$-greedy exploration with $\epsilon=0.2$. After each episode, the policy was updated using Neural Fitted QIteration [16], a batch algorithm that achieves comparatively quick and efficient learning with few observations.

\section{BRAIN-COMPUTER INTERFACE}

The brain-computer interface developed to control the robotic arm is based on movement-related changes in EEG power [15]. We examined both real and imagined movements. Real movements usually elicit more easily detectable brain responses than imagined ones and are thus useful especially in early stages of BCI projects. Imagined movements as a rule produce less pronounced brain activity but might be closer to the signals available in paralyzed patients. Finger tapping (alternatingly touching the thumb with all 4 fingers) and toe clenching were used to elicit EEG responses. The four decoded classes were left hand finger tapping, right hand finger tapping, both toe clenching and the relaxed state where no motor imagery was performed. The commands relayed to the robot controller were related to movement in the following manner: finger tapping navigates the object selection box in the direction of the corresponding hand; toe clenching was used as the confirmation signal for object selection and action trigger. No action was elicited when the relaxed state was decoded.

\section{Signal acquisition}

EEG was acquired at $512 \mathrm{~Hz}$ using two g.USBamp amplifiers in combination with $16 \mathrm{~g}$.SAHARA dry active electrodes (both GUGER TECHNOLOGIES, Graz, Austria). Electrode locations according to the 10-10 system [14] were C1, C3, C5, Fc3, Fc5, Cp3, Cp5, F5, C2, C4, C6, Fc4, Fc6, Cp4, 
Cp6 and F6 with the reference (REF) on the left mastoid and the ground (GND) electrode on the right mastoid. Additionally, electrooculography (EOG) was recorded with four $\mathrm{Ag} / \mathrm{AgCl}$ ring electrodes, two for horizontal and two for vertical eye movements, as well as myoelectrography (EMG) with two $\mathrm{Ag} / \mathrm{AgCl}$ ring electrodes per arm and two for one leg. BCI2000 was used [17] to interface amplifier and signal processing in MATLAB (MathWorks, Natick, MA).

\section{Stimulus presentation}

Prior to the measurement, the subject was instructed to sit as motionlessly as possible and to minimize blinking rate while fixating a fixation point on the screen. During offline trials the subjects were shown arrows pointing left, right or down (in lieu of a confirm icon) for 5 seconds; the interval between the stimuli randomly alternated from $5 \mathrm{~s}$ to $10 \mathrm{~s}$. When only the fixation mark was shown on the screen, the subject had to relax; this defined the rest-state. If either left or right arrows were shown, the subject had to tap or imagine tapping fingers of the corresponding hand. When the down arrow was shown, the subject had to move or imagine moving all his toes in a grabbing way. For each subject, 220 trials per conditions were recorded for both real and imagined movements, resulting in 880 offline trials.

During online trials subjects had to move a cursor from the center of the screen to one of four targets located on the four edges of the screen. The cursor moved to the decoded target in one single step. Left and right hand (imagined) finger tapping was used to move left and right, respectively. Downwards movement was achieved with (imagined) toe movements and upwards movement by relaxing. Again, upward and downward cursor movements were used due to the lack of a visualization for relaxation and confirmation events, respectively. The timing of online trials was as follows. $1 \mathrm{~s}$ rest, followed by $6.5 \mathrm{~s}$ feedback accumulation after which decoding was performed for minimally $2 \mathrm{~s}$ with a timeout of $5 \mathrm{~s}$. If the correct target was hit it was highlighted for $1 \mathrm{~s}$. Timed out trials were not evaluated. For subjects S1-3, 54 trials per condition were recorded for both real and imagined movements, resulting in 216 online trials. Online trials of subjects 4-5 were aborted after fewer trials once it became apparent that no control was possible.

\section{EEG Preprocessing}

First the common average was subtracted from all EEG channels. The signal was then filtered using 6th order high-pass and notch Butterworth filters with a cut-off frequency of $1 \mathrm{~Hz}$ and $48-52 \mathrm{~Hz}$, respectively. Using the MultiTaper method [19], time-resolved spectral magnitude was calculated with a sliding 1-s window and time-steps of $500 \mathrm{~ms}$. Pre-whitening was applied to prevent frequency shifts. The data was then normalized to have 0 mean and a standard deviation of 1 .

Channels for decoding were selected based on the average amplitudes of movement-related responses, i.e., without referring to class differences in order to avoid circularity. To do so the the preprocessed offline trials were cut $3 \mathrm{~s}$ before the start and $5 \mathrm{~s}$ after the end of the stimulus. Baseline removal was achieved by dividing each trial by the mean of the first $500 \mathrm{~ms}$ of all trials. Following, median trials for each condition and channel were calculated. Cp3 and Cp4 showed the strongest alpha band depression during imagined or real hand movement over all subjects. This observation was underpinned by p-values calculated for $8-30 \mathrm{~Hz}$ with the sign test, which were smallest in these channels.

\section{Offline Classification}

To classify rest, left finger, right finger and toe (imagined) movements, the Neural Network Toolbox of MATLAB was used to create an artificial neural network (ANN) with 198 inputs, 20 hidden neurons and 4 outputs. The patternnetfunction, which creates a feed-forward neural network, was used. The neural network was then trained on the 198 previously selected features. Bayesian regulation backpropagation was used as training method. It uses the LevenbergMarquardt optimization, which is known to generate networks that generalize well [10]. The network accuracy was tested using 10-fold cross-validation; p-values for the classification accuracies were calculated against chance level (25\%) using the binomial cumulative distribution function in MATLAB. The four outputs of the network range from -1 to 1 and the one with the highest score was chosen as decoded class.

\section{Online Classification}

In experiments with online control the classification method was identical to its offline counterpart. The ANNs were trained on the whole offline data first and then retrained on 10 trials per condition using the online paradigm before further online testing. In contrast to offline, the same output class had to be decoded four times consecutively as well as having an output score above 0.3 before being accepted. p-values for the classification accuracies were also calculated against chance level (25\%).

\section{RESULTS}

Five healthy subjects (S1-5) with no previous experience with BCI participated in this study, which was approved by the Ethics Committee of the University Medical Center Freiburg and conducted after the subjects gave their written informed consent.

The subjects were two females and three males, all righthanded; their age ranged from 23 to 30 years.

Mean offline decoding accuracy was significantly above chance level (chance $25 \%, p \ll 10^{-16}$ ) for all 5 subjects in both real and imagined movements. For real movements, mean offline accuracy ranged from $36.9 \%$ to $71.8 \%$. For imagined movements, mean online accuracy ranged from $37.0 \%$ to $53.2 \%$. Mean online decoding accuracy was significantly above chance (chance $25 \%, p<10^{-14}$ ) for Subjects $1-3$. Their accuracy ranged from $60.6-74.4 \%$ and from $50.2-$ $60.4 \%$ for real and imagined movements, respectively. Detailed results per subject are shown in Table 1. Median spectra and single trial spectra of electrode $\mathrm{Cp} 3$ for right hand finger tapping and toe clenching of S1 are shown in Figure 3. Even on single trial level, both movements show distinct patterns. Subjects 4 and 5 did not produce accuracies above chance level (chance $25 \%, p>0.1$ ) during the online sessions. 


\begin{tabular}{|c|c|c|c|c|c|c|c|c|c|c|c|c|c|c|}
\hline \multirow[b]{3}{*}{ Subj. } & \multirow[b]{3}{*}{ Sex } & \multirow[b]{3}{*}{ Age } & \multicolumn{6}{|c|}{ Offline } & \multicolumn{6}{|c|}{ Online } \\
\hline & & & & rials & Mean & curacy & & & & rials & Mean & curacy & & \\
\hline & & & real & imag. & real & imag. & real & imag. & real & imag. & real & imag. & real & imag. \\
\hline S1 & $\mathrm{M}$ & 23 & 880 & 880 & $71.8 \%$ & $47.4 \%$ & $<10^{-16}$ & $<10^{-16}$ & 202 & 204 & $61.4 \%$ & $58.8 \%$ & $<10^{-16}$ & $<10^{-16}$ \\
\hline $\mathrm{S} 2$ & M & 26 & 880 & 720 & $63.6 \%$ & $48.2 \%$ & $<10^{-16}$ & $<10^{-16}$ & 213 & 207 & $60.6 \%$ & $60.4 \%$ & $<10^{-16}$ & $<10^{-16}$ \\
\hline S3 & $\mathrm{F}$ & 23 & 30 & 880 & $61.3 \%$ & $53.2 \%$ & $<10^{-16}$ & $<1$ & 211 & 203 & $74.4 \%$ & $50.2 \%$ & $<10^{-16}$ & $<10^{-14}$ \\
\hline S4 & $\mathrm{F}$ & 24 & & 880 & & $37 \%$ & 1 & & 22 & 5 & $\%$ & $28.1 \%$ & 0.59 & 0.31 \\
\hline S5 & M & 30 & 880 & 880 & $44.3 \%$ & $43.1 \%$ & $<10^{-16}$ & $<10^{-16}$ & 57 & 76 & $26.3 \%$ & $30.3 \%$ & 0.42 & 0.15 \\
\hline
\end{tabular}

Table 1. Accuracies and trial numbers per subject. All subjects were right-handed. In the offline setting, the number of trials denotes the trials used for training and validating the classifier, in the online setting the number of test trials.
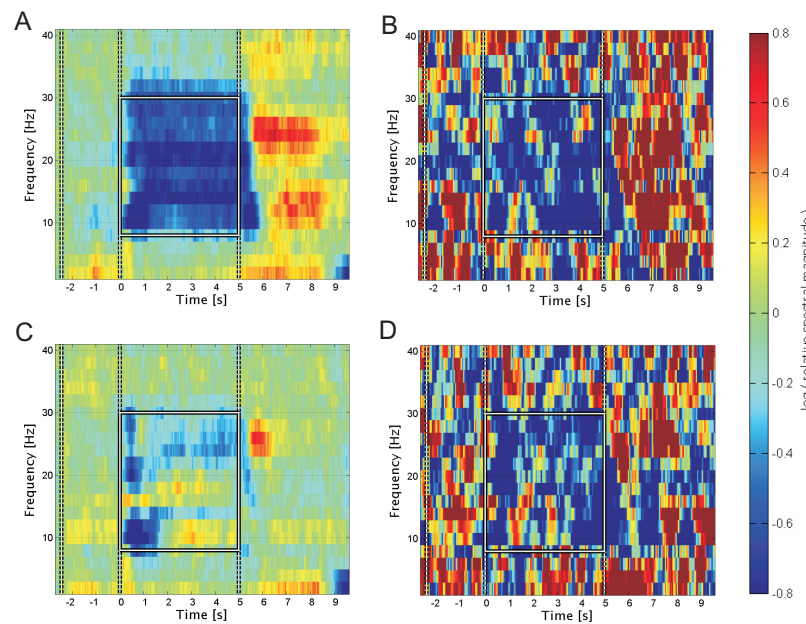

Figure 3. Time-frequency EEG responses for hand and toe movement of S1. All plots show channel Cp3. A: Median of 220 trials, right hand movement. B: Single trial, right hand movement. C: Median of 220 trials, toe movement. D: Single trial, toe movement. The vertical lines indicate the end of the baseline (-2.5 s), stimulus onset $(0 \mathrm{~s})$ and offset $(5 \mathrm{~s})$; the rectangle defines the area which was used to train the ANN $(8-30 \mathrm{~Hz}, 0-5 \mathrm{~s})$.

Subjects 1-3 were subsequently invited to try controlling the Jaco robotic arm based on the same successful online control as described above. Due to the relatively long duration of the entire process (setup, BCI training and robot calibration) subjects tended to become fatigued. As a result, only a limited number of trials with online robot control could be performed for each subject. While this precluded the calculation of accuracy measures, all subjects achieved robot control with a similar success rate as in the previous online cursor control. Despite the limited reliability in the detection of single events, subjects were usually able to successfully grasp the target object due to the ability to simply either amend incorrect commands. In addition, the fact that the selection list wrapped around at the ends enabled them to simply move the opposite direction if one was not recognized properly. Naturally, this also means that there was often no objectively correct action as the users could pursue two paths to the same goal.

\section{DISCUSSION}

In the present study, we have developed and tested an intuitive interface that allows users to convey high-level control signals via a BCI to an intelligent robotic device. Objects detected in a camera image were marked so that they could be selected by the user using motor imagery as commands, and the actual grasp was performed by the autonomous robot controller trained via reinforcement learning. This augmented reality approach proved to be intuitive; all subjects in our study quickly learned to navigate the interface.

Signal detection was robust enough to allow users to grasp intended objects, although dry electrodes were used for greater ease of setup of the system. Movement-related EEG recordings showed the classical suppression in the alpha and beta frequency ranges in a very pronounced manner (Figure 3). We achieved good online and offline decoding in the majority of subjects based on single-trial classification of movementrelated spectral power changes in these frequencies, both during real and imagined upper- and lower-extremity movements. While the accuracy achieved in the present study was not perfect, the main goal of providing subjects with a substantial sense of control was reached.

The results presented here are the first to employ this type of dry electrodes for four-class classification. Comparable electrodes have become commercially available only recently, precluding a direct comparison with other systems using devices that provide markedly different signal quality. State-ofthe-art four class decoding using wet electrodes can achieve higher accuracies between 60-90\%, depending on whether decoding is offline, online, continuous or not, as well as the number of electrodes used [2, 23]. However, the ease-of-use can make up for the reduced accuracy if the focus of a system lies on comfort and rapid deployability like in our case.

Beyond the basic application demonstrated here, this approach can be extended in various respects. On the robotic side, different grasp types may be inferred for one object and presented in a symbolic form, replacing the simple selection box in the current study. Furthermore, other robotic systems like mobile and/or humanoid robots could be integrated in the setup to share tasks. Preliminary work in our labs has recently been successful at using a mobile humanoid robot for a fetch-and-carry task.

On the EEG side, our focus thus far has been on establishing a comfortable and quickly usable setup, rather than optimizing the decoding accuracy. Although having achieved good online and offline decoding in the majority of subjects, decoding performance can likely be further increased using a range of established EEG interfacing techniques, such as sophisticated channel and feature selection, spatial filtering, and adaptive decoding [13, 9, 20]. A further performance boost can be expected from moving to intracranial (invasive) control signals, such as the electrocorticogram (ECoG) recoded 
from the brain surface, intracortical local field potentials, or single-neuron activity $[22,11]$. Although invasive measurements are usually associated with low-level paradigms, their robustness and high accuracy make them very interesting signal sources for high-level BCIs. Whether high-level control would still provide an advantage with them remains to be investigated in future work. It is also worth noting that the remote nature of the system would be even more justified, since there are restrictions on using robotic equipment in the vicinity of implanted patients.

A noteworthy feature of the BCI system used in our study is that it combined three (potentially) adaptive entities: the users brain, the decoder, and the autonomous agent. Firstly, to train the autonomous agent, techniques of both supervised and reinforcement learning were used (see Methods), and could be employed to adapt behaviors if conditions of the system or requirements change, such as in case of damage to the hardware. Secondly, although the employed decoder was not continually adapting, it was updated based on new data from each online session before starting the next session, thus effectively adapting on the time scale corresponding to the duration of individual experimental sessions. And finally, although learning and adaptivity on the users side was not assessed in the present study, there is a large and growing body of evidence that learning on the neuronal level occurs during BCI control and is essential for its success [21]. An interaction between these three adaptive systems leads to potentially interesting co-adaptivity scenarios that go beyond the dual coadaptivity problems previously addressed in the BCI context.

In summary, here we have presented a novel BCI setup that is centered on an intuitive user interface based on an augmented reality approach. This interface links signals derived from dry-electrode EEG recordings to control an intelligent robotic device for reaching and grasping. While not meant to replace approaches aimed at high accuracy, such as systems based on intracranial signals, the setup proved to be very useful for extensive testing and optimization of the user interface. This platform is well suited for the development of clinical BCI systems in which interface properties have to be tailored to patient's needs and their individual environment.

\section{ACKNOWLEDGMENT}

This work was partly supported by the BrainLinksBrainTools Cluster of Excellence funded by the German Research Foundation (DFG, grant number EXC 1086) and by the German Federal Ministry of Education and Research grant 16SV5834 NASS to the University of Freiburg. We also thank the anonymous reviewers for their useful suggestions which helped improve the final paper.

\section{REFERENCES}

1. Allison, B. Z., Leeb, R., Brunner, C., Müller-Putz, G. R., Bauernfeind, G., Kelly, J. W., and Neuper, C. Toward smarter BCIs: extending BCIs through hybridization and intelligent control. Journal of Neural Engineering 9 (2012), 1.

2. Brunner, C., Naeem, M., Leeb, R., Graimann, B., and Pfurtscheller, G. Spatial filtering and selection of optimized components in four class motor imagery EEG data using independent components analysis. Pattern Recognition Letters 28, 8 (2007), 957-964.
3. Bryan, M., Green, J., Chung, M., Chang, L. Y., Scherer, R., Smith, J. R., and Rao, R. P. N. An adaptive brain-computer interface for humanoid robot control. In Humanoids (2011), 199-204.

4. Collinger, J. L., Wodlinger, B., Downey, J. E., Wang, W., Tyler-Kabara, E. C., Weber, D. J., McMorland, A. J. C., Velliste, M., Boninger, M. L., and Schwartz, A. B. High-performance neuroprosthetic control by an individual with tetraplegia. The Lancet 381, 9866 (2013), 557-564.

5. Escolano, C., Antelis, J., and Minguez, J. Human brain-teleoperated robot between remote places. In Robotics and Automation, 2009. ICRA '09. IEEE International Conference on (2009), 4430-4437.

6. Ferreira, A., Bastos-Filho, T., Sarcinelli-Filho, M., Cheein, F., Postigo, J., and Carelli, R. Teleoperation of an industrial manipulator through a tcp/ip channel using eeg signals. In Industrial Electronics, 2006 IEEE International Symposium on, vol. 4 (2006), 3066-3071.

7. Hochberg, L. R., Bacher, D., Jarosiewicz, B., Masse, N. Y., Simeral, J. D., Vogel, J., Haddadin, S., Cash, J. L. S. S., van der Smagt, P., and Donoghue, J. P. Reach and grasp by people with tetraplegia using a neurally controlled robotic arm. Nature 485, 7398 (2012), 372-375.

8. Lampe, T., and Riedmiller, M. Acquiring visual servoing reaching and grasping skills using neural reinforcement learning. IEEE International Joint Conference on Neural Networks (IJCNN) (2013).

9. Lotte, F., Congedo, M., Lcuyer, A., Lamarche, F., and Arnaldi, B. A review of classification algorithms for EEG-based brain-computer interfaces. Journal of Neural Engineering 4, 2 (2007), R1-R13.

10. MacKay, D. J. C. A practical bayesian framework for backpropagation networks. Neural Computation 4, 3 (1992), 448-472.

11. Moran, D. Evolution of brain-computer interface: action potentials, local field potentials and electrocorticograms. Current Opinions in Neurobiology 20, 6 (2010), 741-745.

12. Musallam, S., Corneil, B. D., Greger, B., Scherberger, H., and Andersen, R. A. Cognitive control signals for neural prosthetics. Science 305 (2004), 258-262.

13. Neuper, C., Müller-Putz, G. R., Scherer, R., and Pfurtscheller, G. Motor imagery and EEG-based control of spelling devices and neuroprostheses. Progress in Brain Research 159 (2006), 393-409.

14. Oostenveld, R., and Praamstra, P. The five percent electrode system for high-resolution EEG and ERP measurements. Clinical Neurophysiology 112, 4 (2001), 713-719.

15. Pfurtscheller, G., and F.H. Lopes da Silva. Event-related EEG/MEG synchronization and desynchronization: basic principles. Clinical Neurophysiology 110, 11 (1999), 1842-1857.

16. Riedmiller, M. Neural fitted Q iteration - first experiences with a data efficient neural reinforcement learning method. In European Conference on Machine Learning, Springer (2005), 317-328.

17. Schalk, G., Mcfarl, D. J., Hinterberger, T., Birbaumer, N., and Wolpaw, J. R. BCI2000: A general-purpose brain-computer interface (BCI) system. IEEE Transactions on Biomedical Engineering 51 (2004), 2004.

18. Sutton, R. S., and Barto, A. G. Reinforcement Learning: An Introduction (Adaptive Computation and Machine Learning). A Bradford Book, 1998.

19. Thomson, D. Spectrum estimation and harmonic analysis. Proceedings of the IEEE 70, 9 (1982), 1055-1096.

20. Vidaurre, C., Kawanabe, M., von Bünau, P., Blankertz, B., and Müller, K. R. Toward unsupervised adaptation of LDA for brain-computer interfaces. IEEE Transactions on Biomedical Engineering 58, 3 (2011), 587-597.

21. Vidaurre, C., Sannelli, C., Müller, K. R., and Blankertz, B. Machine-learning-based coadaptive calibration for brain-computer interfaces. Neural Computation 16 (2010).

22. Waldert, S., Pistohl, T., Braun, C., Ball, T., Aertsen, A., and Mehring, C. A review on directional information in neural signals for brain-machine interfaces. Journal of Physiology - Paris 103, 3-5 (2009), 244-254.

23. Wang, D., Miao, D., and Blohm, G. Multi-class motor imagery EEG decoding for brain-computer interfaces. Frontiers in Neuroprosthetics 6 (2012), 151. 